\title{
Challenges in basic life support and automated external defibrillator training of deaf individuals
}

\author{
Matej Strnad ${ }^{1,2,3, \star}$, Zdenko Šalda ${ }^{4,5}$, Boštjan Jerko ${ }^{6}$, Vida Vrečar ${ }^{1}$, \\ Vesna Borovnik Lesjak ${ }^{1}$, Rok Petrovčič ${ }^{2}$
}

\author{
${ }^{1}$ Center for Emergency Medicine, \\ Prehospital unit, Community healthcare \\ center, Cesta proletarskih brigad 21, \\ 2000 Maribor, Slovenia \\ ${ }^{2}$ Emergency department, University \\ Medical Center Maribor, Ljubljanska ul. \\ 5, 2000 Maribor, Slovenia \\ ${ }^{3}$ Department of Emergency Medicine, \\ Medical faculty, University of Maribor \\ Taborska 8, 2000 Maribor, Slovenia \\ ${ }^{4}$ Faculty of Health Sciences, University of \\ Novo Mesto, Na Loko 2, 8000 Novo \\ mesto, Slovenia \\ ${ }^{5}$ Community Health Center Trebnje, \\ Golijev trg 3, 8210 Trebnje, Slovenia \\ ${ }^{6}$ Japina LLC, Ravnik 31, 8232 Šentrupert, \\ Slovenia
}

\section{*Correspondence}

strnad.matej78@gmail.com

(Matej Strnad)

\begin{abstract}
Objectives: Basic life support (BLS) with the use of an automated external defibrillator (AED) is linked to survival of patients with out-of-hospital cardiac arrest (OHCA). However, the BLS protocol is not tailored to specific needs of the deaf who encounter many challenges during BLS training.

Methods: The BLS and AED protocol was modified according to the challenges faced by deaf people. Pre-course BLS and AED knowledge was tested using a questionnaire. After completion of a practical course, each participant was presented with an OHCA scenario using a manikin. Qualitative and quantitative data on BLS and AED performance were collected with a modified Cardiff test and the QCPR mobile application. Results of the knowledge test and performance scores are presented with values and frequencies. Correlations between pre- and post-course BLS and AED knowledge and performance were analyzed and presented with Spearman's rho.

Results: 51 deaf volunteers from seven Slovenian deaf associations participated in the study. The pre-course knowledge test scores were 3.5 points out of 10 and considered low. The rest of the results were also poor. BLS performance using the modified Cardiff test post-course was as follows: $52.9 \%$ of the participants used a safe approach, $58.8 \%$ checked responsiveness and $51.0 \%$ sent a text message to the rescue service. Only $43.1 \%$ opened the airway and $49.0 \%$ checked initial breathing. $80.4 \%$ of deaf rescuers performed chest compressions on the lower half of the sternum and $52.9 \%$ compressed with adequate depth. According to the QCPR application the best performance was achieved with a compression score of $61.1 \%$ and flow fraction $74.9 \%$.

Conclusions: This study shows that a comprehensive and assiduous approach is needed for effective BLS and AED training courses for deaf individuals.
\end{abstract}

\section{Keywords}

Automated external defibrillator; Cardiopulmonary resuscitation; Deafness; Education

\section{Introduction}

An out-of-hospital cardiac arrest (OHCA) is one of the leading causes of death in Europe [1] and the recommended treatment for an OHCA are immediate basic life support procedures (BLS) [2]. Teaching lay people BLS is essential in survival of patients with OHCA [3]. However, guidelines for BLS are not tailored to specific needs of the deaf. There are several steps that deaf individuals have difficulties with or cannot perform: listening to check initial breathing, calling the emergency number 112 or using an automated external defibrillator (AED) with voice instructions [4]. The use of an AED is one of the basic resuscitation procedures to stop malignant arrhythmias [5], but the device itself is often not adapted for use by the deaf, as it provides only voice instructions. The AED must be equipped with visual instructions to shorten the time to defibrillation [6]. To the best of our knowledge, few have studied the BLS performance by deaf individuals. Sandroni et al. [6] assessed the capability of deaf rescuers to defibrillate effectively using an AED with visual prompts and Tomasetti et al. [7] evaluated the cognitive and psychomotor scores immediately after the course.

Most deaf people use sign language with its own grammar and syntax. It differs markedly from the mother tongue and is not its gestured representation [8]. Therefore, the deaf who use sign language are not fluent in the language of their surroundings leading to low levels of reading comprehension [9]. In addition, low health literacy and knowledge are described in deaf people [10-12]. They are not familiar with the symptoms [10] and vocabulary regarding most common cardiovascular health issues [13]. Therefore, both deaf trainees and their instructors face many challenges during BLS training, from communication issues to inability to follow standard BLS protocols. The aim of the study was to assess hindrances the 
TA B L E 1. Analysis of questions on pre-course knowledge test $(\mathrm{N}=51)$.

Question $\%$ Correct answers $(\mathrm{N})$

1. How do you recognize a person in cardiac arrest? $43.1 \%(22)$

2. Who can help in a case of cardiac arrest?

3. A person suddenly loses consciousness and collapses. What do you do?

$33.3 \%(17)$

4. How do you check if a person is breathing normally?

$47.1 \%(24)$

5. What kind of breathing is NOT a sign of life?

$33.3 \%(17)$

6. How is basic life support correctly performed?

$25.5 \%(13)$

7. On the sketch of the torso below mark with a cross the correct site for chest compressions during $43.1 \%(22)$ basic life support

8. How do you perform artificial breaths in an unconscious person?

9. What do you do if you are unsure whether a person is in cardiac arrest or not?

$39.2 \%(20)$

10. What is an AED (automatic external defibrillator)?

$29.4 \%(15)$

deaf population faces in regard to BLS and AED protocols and to modify them accordingly. We measured the effectiveness of the modified BLS training for the deaf by evaluating cardiopulmonary resuscitation (CPR) performance. During the course, we identified specific challenges deaf adults face during BLS training and proposed measures to improve BLS and AED courses for the deaf.

\section{Methods}

The study was approved by the National Medical Ethics Committee of the Republic of Slovenia (No. 0120-541/2017/6). Before the beginning of the course, participants received a letter describing the purpose and content of the study. An informed consent and a data administration consent form were completed by participants consistent with the Global Data Protection Regulation (EU) 2016/679. Explanation about the study was provided by the research coordinator. All the information was translated into sign language by a certified sign interpreter.

\subsection{Study design}

In the first step, an occupational medicine specialist (Z $\breve{S})$ analyzed the BLS and AED protocol from the latest European Resuscitation Council Guidelines [2] and modified it according to anticipated challenges the deaf would face. The following modifications were proposed to enable successful BLS and AED training of deaf individuals: BREATHING look and feel for normal breathing; UNRESPONSIVE AND NOT BREATHING NORMALLY - ask a helper to call the emergency services or activate them yourself by sending a text message with crucial data to the Regional Emergency Notification Center -112, which transmits the message to the Emergency medical dispatch Center or informs the deaf call center (available 24 hours daily) and asks them to call 112; WHEN AED ARRIVES- switch on the AED, put it into the visual field and follow the visual prompts.

In the second step, a practical course for deaf volunteers was conducted. In each group, there were a maximum of 10 participants, 5 per instructor. At the beginning of the course, each participant filled out a pre-course BLS and AED knowledge test [14]. After the pre-course test, there was a 30minute theoretical lecture and a 45-minute practical training in the modified BLS and AED protocol led by a physician accompanied by a certified sign interpreter. Each participant practiced with a cardboard AED prop with adhesive electrode pads on their own torso training manikin (Prestan CPR Torso, Erler Zimmer GmbH \& Co. KG, Lauf, Germany). An AED trainer with visual prompts (AED Trainer, Defibtech LLC, Guilford, USA) was used for guidance. Each trained participant was asked to solve an OHCA scenario on a manikin (Little Anne QCPR, Laerdal Medical, Stavanger, Norway) using the AED trainer. Each OHCA scenario lasted 2 minutes and each participant performed one BLS cycle. To evaluate practical skills gained during the course, qualitative and quantitative data on BLS and AED performance were collected with a modified Cardiff test and the QCPR mobile application (QCPR training 4.0.0. by Laerdal Medical, Stavanger, Norway). The Cardiff test was modified to match the modifications for the deaf made to the BLS and AED protocol. Items of both instruments were collected on a joint checklist. Incorrect performance of the task was scored as 1 point, whereas partially correct or correct performance was scored with 2 to 4 points. Additionally every item was graded as correct or incorrect. BLS performance was evaluated by one of the two instructors

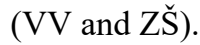

\subsection{Statistical analysis}

Frequencies are reported to describe sociodemographic characteristics and answers on the pre-course knowledge test and post-course performance analysis. Practical performance analyzed by the QCPR Laerdal mobile application is presented as means with standard deviation. Correlations among variables were tested using Spearman's rho. SPSS software (version 25, SPSS Inc., Chicago, IL, USA) was used for statistical analysis.

\section{Results}

\subsection{Sample}

We contacted all 13 Slovenian deaf associations by email and invited them to participate in the study. We asked them 
TA B L E 2. Performance of individual tasks of BLS and AED steps on the modified Cardiff test $(\mathbf{N}=\mathbf{5 1})$.

\begin{tabular}{|c|c|c|}
\hline BLS Step & BLS Task & $\%$ Performed Correctly $(\mathrm{N})$ \\
\hline Step 1: Safety & Safe approach & $52.9 \%(27)$ \\
\hline \multirow{2}{*}{ Step 2: Responsiveness } & Check responsiveness - shake & $58.8 \%(30)$ \\
\hline & Indicate looking for help & $29.4 \%(15)$ \\
\hline \multirow[t]{3}{*}{ Step 3: Airway and breathing } & Initial airway opening & $43.1 \%(22)$ \\
\hline & Initial check/clear airway & $49.0 \%(25)$ \\
\hline & Initial breathing check & $31.4 \%(16)$ \\
\hline \multirow[t]{2}{*}{ Step 4: Call for help } & Send SMS to rescue service & $51.0 \%(26)$ \\
\hline & Send someone to find help and AED & $31.4 \%(16)$ \\
\hline \multirow[t]{6}{*}{ Step 5: CPR sequence } & Hand position & $80.4 \%(41)$ \\
\hline & Average number of compressions (100-120/min) & $37.3 \%(19)$ \\
\hline & Average depth of compressions & $52.9 \%(27)$ \\
\hline & Open airway for rescue breaths & $43.1 \%(22)$ \\
\hline & Close the nose & $66.7 \%(34)$ \\
\hline & Perform rescue breaths, check for moving of the thorax & $33.3 \%(17)$ \\
\hline \multirow[t]{5}{*}{ Step 6: AED usage } & Open and turn on AED & $84.3 \%(43)$ \\
\hline & AED electrode on right position & $92.2 \%(47)$ \\
\hline & AED in visual field & $78.4 \%(40)$ \\
\hline & Hands-off check during analysis & $64.7 \%(33)$ \\
\hline & Check for safety and push shock button & $45.1 \%(23)$ \\
\hline Step 7: & Continue with BLS & $72.5 \%(37)$ \\
\hline
\end{tabular}

to forward our invitation to their members. Deafness was defined as hearing loss of more than $95 \%$ by Fowler and using sign language for communication. 51 volunteers from 7 deaf associations participated in the study from 30 January 2019 to 15 January 2020. A total of 28 (55\%) were males and $23(45 \%)$ were female. Mean age was 53.6 years of age. Most (78\%) a BLS and AED course in the past, usually as part of the driving license course $(27 \%)$, a free public course $(19 \%)$ or either at school or at work (16\% each). Mean educational level was 4 (ranging from 1 - unfinished primary school to 8 - Master of Arts/Master of Science) an equivalent of 3 years of vocational secondary education (43\%). Fifteen participants (29\%) had a healthcare worker in the family.

\subsection{Pre-course}

The mean sum of correct answers on the pre-course knowledge test was $3.51( \pm 2.22$; range $0-8$ with max 10) Percentages of correct answers for each question are presented in Table 1 .

\subsection{Post-course}

The mean score on the post-course modified Cardiff test was 42.16 ( \pm 7.22 ; range 28-55).

Percentages of correctly performed BLS and AED tasks are presented in Table 2. Score range, means with standard deviation, minimum and maximum scores achieved on individual BLS steps on the modified Cardiff test are presented in Table A in Supplementary material.

A general overview of BLS performance recorded with the QCPR application with quality assessment scores was as fol- lows: the overall score (based on compressions, ventilations, and flow fraction) was $53.8 \%$ with a flow fraction of $75 \%$, compression score of $61 \%$ and ventilation score of $41 \%$.

Correct performance of individual tasks of chest compressions and ventilations assessed by the QCPR application is presented in Table 3.

Analysis of correlations between sociodemographic characteristics of the sample, pre-course knowledge of BLS and AED, and post-course BLS and AED awareness gain is presented in Table 4. Younger age and higher education level are significantly associated with higher score on pre-course test. Presence of a healthcare worker is significantly associated with the overall score on the QCPR application.

\section{Discussion}

\subsection{BLS performance}

The current study revealed that mere adjustments of the BLS and AED protocol originally designed for hearing people do not suffice for effective training of deaf individuals. Three crucial tasks of the BLS and AED protocol were modified as the first step of the study: breathing check, alerting emergency services and using an AED. Similar limitations were pointed out in research by Unnikrishnan et al. [4].

In the present study, BLS and AED knowledge was tested before the course using a previously developed questionnaire for schoolchildren [14]. The results suggest that BLS knowledge by the deaf people is poor (an average score of 3.5/10) compared to hearing peers (7.8/10) [15] and schoolchildren (an 
TA B L E 3. Qualitative analysis of BLS performance with QCPR application $(\mathbf{N}=51)$.

\begin{tabular}{|llc|}
\hline BLS Step & BLS Task & \% Performed Correctly (N) \\
\hline Chest Compressions & Average rate 100-120/min & $41.2 \%(21)$ \\
& $100 \%$ correct chest compressions & $2.0 \%(1)$ \\
& Average depth 50-60 mm & $23 \%(12)$ \\
\hline Tetal number: $140-190$ & $23.5 \%(12)$ \\
\hline Fentilations & Flow fraction $>70 \%$ & $47.1 \%(24)$ \\
\hline & Total number: 12 & $2.0 \%(1)$ \\
\hline & Ventilations with adequate chest rise: $>50 \%$ & $49.0 \%(25)$ \\
\hline
\end{tabular}

TA B L E 4. Correlation coefficients between baseline characteristics, pre-course test and post-course scores.

\begin{tabular}{lccc} 
Gender & $\begin{array}{c}\text { Sum of correct answers Sum of correct answers } \\
\text { on pre-course test }\end{array}$ & $\begin{array}{c}\text { on Cardiff } \\
\text { Overall score on QCPR }\end{array}$ & -0.095 \\
Age & -0.068 & 0.042 & 0.01 \\
Education & $-0.385^{* *}$ & -0.102 & 0.225 \\
Healthcare worker in family & $0.452^{* *}$ & 0.24 & $0.325^{*}$ \\
Sum of correct answers on pre-course test & 0.021 & -0.104 & 0.11 \\
\hline
\end{tabular}

Note: **, correlation significant at the 0.01 level (2-tailed); *, correlation significant at the 0.05 level (2-tailed).

average score of 6.0/10, data not published yet). The most incorrect answers were given to the basic questions representing the core of BLS, supporting the observation of insufficient BLS and AED knowledge by the deaf.

Our study showed that BLS and AED performance scored and assessed by the modified Cardiff test was also poor compared to lay people who were given the instructor-led BLS training (76.7\% vs $87.0 \%$, respectively) [16]. In our observation, merely half of the participants would use a safe approach to the OHCA victim, check responsiveness, and send a text message to the rescue service. Less than half of them would open the airway and check initial breathing or send someone for help or for an AED. Regarding chest compressions, more than $80 \%$ of participants would perform chest compressions in the correct position, $53 \%$ of them compressing one third of the diameter of the chest, but less than half of them at an adequate rate. Better results were observed by Tomasetti et al. [7] where deaf individuals participated in the standard American National Red Cross 4-hour course signed by the course instructor achieved $27 / 32$ points $(84 \%)$ on an immediate posttest performance score. Slightly better results were yielded by deaf students given the same 4-hour course who were also shown a captioned video with CPR instructions or a video with CPR instructions interpreted into sign language by the course instructor ( $86 \%$ and $84 \%$, respectively). In contrast to Tomasetti et al. [7], a 75-minute course was given to the participants in the present study. In addition, there were some differences in the study population (college students compared to participants with low average educational level and higher age in our study) which could also contribute to the better results in the study by Tomasetti et al. [7].

The best performance scores yielded on the manikin in the present study were the compression score and flow frac- tion representing "low-flow" state in cardiac arrest. Overall performance score on the manikin was reduced on account of poorer results in the ventilation score. There were some score discrepancies in BLS performance between the modified Cardiff test and feedback data from the manikin. According to the manikin data, only $23 \%$ of participants compressed the chest with the correct average depth of 50 to $60 \mathrm{~mm}$ compared to $53 \%$ according to the modified Cardiff test. This fact could be due to more accurate and sophisticated measurements provided by the manikin software, whereas the compression depth on the modified Cardiff test was subjective and estimated by observation.

Family conversations about family medical history and other incidental sources of health knowledge are crucial for developing strong health literacy skills [12, 13]. In the present study a family member working as a healthcare provider was associated with better results in the overall QCPR score after the course. It is likely that a healthcare worker stimulates the conversation with other family members about the medical issues and through family communication promotes an interest in a healthy life style including attaining BLS skills.

\subsection{AED}

Using an AED can be a challenge for the deaf, as many AEDs provide only voice prompts [17]. On the other hand, untrained deaf rescuers are capable of using an AED with visual prompts appropriately after basic training [6]. Only four participants in our study failed to attach the AED pads in a correct position and eight of them forgot to turn it on. Despite the fact that nearly $80 \%$ of deaf participants put the AED into their visual field to be guided by visual instructions, $55 \%$ of the participants failed to successfully perform the safety check and press the shock button. Sandroni et al. described that $22 \%$ of participants did 
not deliver a shock because they expected the defibrillator to do it automatically [6].

\subsection{Challenges during the BLS course}

Communication is the basic challenge for educators of deaf individuals. The major differences between BLS instructions for deaf and non-deaf are the need for a sign interpreter, the need to modify the terminology of BLS instructions and careful explanation of terminology [18]. During the pre-test we observed an extensive effort from the sign language interpreter to explain the meaning and the purpose of the questions. This observation and the low score on the pre-test could be due to a low level of reading comprehension by the deaf. It has been shown that an average student with hearing loss graduates from high school with reading comprehension skills at about fourth grade level [9].

In addition, there is growing evidence in the literature regarding health literacy weaknesses by the deaf $[10,11]$. Findings from several studies indicate that deaf individuals have weaker functional health literacy and a smaller fund of cardiovascular health knowledge [10-12]. Nearly $40 \%$ of the deaf could not list any of the most common symptoms of a heart attack, while over $60 \%$ could not list a single stroke symptom $[10,13]$. Moreover, more than one third of the deaf would not call the emergency medical number if they thought they were having a heart attack or stroke, thinking that it is not deaf-accessible [10]. This observation is similar to our study where half of the participants would not send a message to the rescue service in the case of an OHCA even though it could be activated through a text message. Although neither reading comprehension nor health literacy were analyzed in the present study, we assume that low level of both in our study group could contribute to the low scores on the pre-test.

Deficits in reading comprehension and low level of health literacy have an impact on the BLS course. Presentation designed for deaf adults should use simpler English grammar and vocabulary, and more visual information [12]. Our BLS course was led by a physician accompanied by a sign language interpreter from a non-medical field. Signed interpretation appears to be the better way to communicate BLS information to the deaf learners. Signed interpretation may eliminate poor reading comprehension as a potential barrier in learning and retention of BLS skills [7]. Other possible helpful additions could be avoiding changing the position of the instructor during the presentation as it distracts the deaf participants. This is due to the enhanced peripheral visual attention $[19,20]$ making the deaf subjects more susceptible to peripheral distracters [21]. Also, addressing the short attention span of deaf participants is important. During the course, the attention span of the participants was shorter than expected (it lasted between 25-30 minutes). This observation is supported by studies reporting that poor sustained attention in deaf children improved little with increasing years [22, 23].

\subsection{Suggestions}

Based on observations from our study the following adjustments for BLS and AED training for the deaf are suggested: presentations and tests should be designed for deaf adults using simpler English grammar and vocabulary. More visual information (including graphics/videos) should be included in the course to yield better results (like in the study by Tomasetti et al. [7]). The terminology of BLS instructions should be modified and simplified to a lower level of comprehension. The entire course should be prepared in shorter segments and accompanied by a sign interpreter. Peripheral visual distractors should be minimized during the training (an AED should be put into the visual field).

\subsection{Limitations of the study}

The study has several limitations. Firstly, we had a small sample size. Secondly, the pre-test was designed for schoolchildren and not for deaf individuals. Due to reading comprehension and health literacy issues a specific pre-test should be developed for deaf adults. Thirdly, we used different measuring instruments before and after the course which made comparison of the results before and after an intervention difficult. Furthermore, the duration of the course was short and a 4-hour training with additional visual aids may result in better post-course scores. Finally, we have not tested the retention of BLS and AED skills.

\section{Conclusions}

This study shows that a more comprehensive approach is needed in BLS and AED training for the deaf. Challenges in this specific population require specific adjustments of BLS and AED courses, extending beyond modifications of the BLS algorithm. An approach in BLS training focusing only on crucial interventions during CPR (chest compressions only guidelines for the deaf performing CPR) should be considered and evaluated. Further studies are needed to determine an effective approach to BLS and AED training courses for the deaf.

\section{AUTHOR CONTRIBUTIONS}

MS has contributed in the conception and design of the work, acquisition, analysis and interpretation of the data and has substantively revised the drafted work. Z $\breve{S}$ has contributed substantially to the conception and design of the work, the acquisition and interpretation of the data. BJ has contributed in the design of the work, acquisition, analysis and interpretation of the data. VBL contributed to the design of the work, analysis and interpretation of the data and has substantively revised the drafted work. VV has contributed substantially in acquisition, analysis and interpretation of the data. RP has contributed in the conception and design of the work, acquisition and interpretation of the data and has revised the drafted work. All authors read and approved the final manuscript.

\section{ETHICS APPROVAL AND CONSENT TO PARTICIPATE}

The study was approved by the National Medical Ethics Committee of the Republic of Slovenia (No. 0120-541/2017/6). 


\section{ACKNOWLEDGMENT}

We would like to thank all participating deaf associations in Slovenia for making the sampling possible.

\section{FUNDING}

Design of the study, collection, analysis, and interpretation of data and writing the manuscript was done by the authors without any funding sources to be declared.

\section{CONFLICT OF INTEREST}

The authors declare that there is no conflict of interest regarding the publication of this article.

\section{SUPPLEMENTARY MATERIAL}

Supplementary material associated with this article can be found, in the online version, at https: //oss.signavitae.com/mre-signavitae/article/ $1355058895624519680 /$ attachment/SV2020120804_ Supplementary\%20material.pdf.

\section{DATA AVAILABILITY}

The data used to support the findings of this study are available from the corresponding author upon request.

\section{REFERENCES}

[1] Gräsner JT, Lefering R, Koster RW, Masterson S, Böttiger BW, Herlitz $\mathrm{J}$, et al. EuReCa ONE-27 Nations, ONE Europe, ONE Registry: a prospective one month analysis of out-of-hospital cardiac arrest outcomes in 27 countries in Europe. Resuscitation 2016; 105: 188-195.

[2] Perkins GD, Handley AJ, Koster RW, Castrén M, Smyth MA, Olasveengen $\mathrm{T}$, et al. European resuscitation council guidelines for resuscitation 2015: section 2. Adult basic life support and automated external defibrillation. Resuscitation. 2015; 95: 81-99.

[3] Greif R, Lockey AS, Conaghan P, Lippert A, De Vries W, Monsieurs KG. European resuscitation council guidelines for resuscitation 2015: section 10. Education and implementation of resuscitation. Resuscitation. 2016; 95: 288-301.

[4] Unnikrishnan R, Babu AS, Rao PT, Aithal V, Krishna HM. Training individuals with speech and hearing impairment in basic life support: a pilot study. Resuscitation. 2017; 117: e23-e24.

[5] Kerber RE, Becker LB, Bourland JD, Cummins RO, Hallstrom AP, Michos MB, et al. Automatic external defibrillators for public access defibrillation: recommendations for specifying and reporting arrhythmia analysis algorithm performance, incorporating new waveforms, and enhancing safety. a statement for health professionals from the American Heart Association Task Force on Automatic External Defibrillation, Subcommittee on AED Safety and Efficacy. Circulation. 1997; 95: 16771682.

[6] Sandroni C, Fenici P, Franchi ML, Cavallaro F, Menchinelli C, Antonelli
M. Automated external defibrillation by untrained deaf lay rescuers. Resuscitation. 2004; 63: 43-48.

[7] Tomasetti JA, Beck KH, Clearwater HE. An analysis of selected instructional methods on cardiopulmonary resuscitation retention competency of deaf and non-deaf college students. American Annals of the Deaf. 1983; 128: 474-478.

[8] Valli C, Lucas C, Mulrooney K. Linguistic of American sign language. 4 ed. Washington DC: Gallaudet University Press. 2005.

[9] Holt JA. Stanford achievement test-8th edition: reading comprehension subgroup results. American Annals of the Deaf. 1993; 138: 172-175.

[10] Margellos-Anast H, Estarziau M, Kaufman G. Cardiovascular disease knowledge among culturally deaf patients in Chicago. Preventive Medicine. 2006; 42: 235-239.

[11] Pollard RQ, Barnett S. Health-related vocabulary knowledge among deaf adults. Rehabilitation Psychology. 2009; 54: 182-185.

[12] Smith SR, Samar VJ. Dimensions of deaf/hard-of-hearing and hearing adolescents' health literacy and health knowledge. Journal of Health Communication. 2016; 21: 141-154.

[13] Smith SR, Kushalnagar P, Hauser PC. Deaf adolescents' learning of cardiovascular health information: sources and access challenges. Journal of Deaf Studies and Deaf Education. 2016; 20: 408-418.

[14] Borovnik Lesjak V, Šorgo A, Strnad M. Development, validation and assessment of the test on knowledge about basic life support and use of automated external defibrillator among schoolchildren. Scandinavian Journal of Trauma, Resuscitation and Emergency Medicine. 2019; 27: 114.

[15] Rihtar A. Level of knowledge of basic resuscitation procedures with the use of the automated external defibrillator at lay persons before and after 2-hour education. Maribor: University of Maribor. 2019. (In Slovene).

[16] Bylow H, Karlsson T, Claesson A, Lepp M, Lindqvist J, Herlitz J. Selflearning training versus instructor-led training for basic life support: a cluster randomised trial. Resuscitation. 2019; 139: 122-132.

[17] Müller MP, Poenicke C, Kurth M, Richter T, Koch T, Eisold C, et al. Quality of basic life support when using different commercially available public access defibrillators. Scandinavian Journal of Trauma, Resuscitation and Emergency Medicine. 2015; 23: 48.

[18] Beck KH, Tomasetti JA. A national survey of cardiopulmonary resuscitation training for the deaf. American Annals of the Deaf. 1983; 128: 909-912.

[19] Bavelier D, Tomann A, Hutton C, Mitchell T, Corina D, Liu G, et al. Visual attention to the periphery is enhanced in congenitally deaf individuals. The Journal of Neuroscience. 2000; 20: RC93.

${ }^{[20]}$ Dye MW, Hauser PC, Bavelier D. Is visual selective attention in deaf individuals enhanced or deficient? The case of the useful field of view. PLoS ONE. 2009; 4: e5640.

[21] Dye MWG, Baril DE, Bavelier D. Which aspects of visual attention are changed by deafness? The case of the attentional network test. Neuropsychologia. 2007; 45: 1801-1811.

[22] Dye MWG, Hauser PC. Sustained attention, selective attention and cognitive control in deaf and hearing children. Hearing Research. 2014; 309: 94-102.

[23] Horn DL, Davis RAO, Pisoni DB, Miyamoto RT. Development of visual attention skills in prelingually deaf children who use cochlear implants. Ear and Hearing. 2005; 26: 389-408.

How to cite this article: Matej Strnad, Zdenko Šalda, Boštjan Jerko, Vida Vrečar, Vesna Borovnik Lesjak, Rok Petrovčič. Challenges in basic life support and automated external defibrillator training of deaf individuals. Signa Vitae. 2021;17(2):98-103. doi:10.22514/sv.2021.019. 